\title{
Adaptive Capacity to Extreme Heat: Results from a Household Survey in Houston, Texas ${ }^{\mathscr{O}}$
}

\author{
Mary H. Hayden, ${ }^{a}$ Olga V. Wilhelmi, ${ }^{\text {a }}$ Deborah BanerJee, ${ }^{\text {b Tamara Greasby }},{ }^{c}$

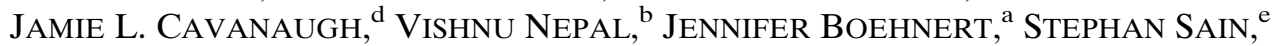 \\ CRYSTAL BURGHARDT, ${ }^{\mathrm{f}}$ AND STEPHANIE GOWER ${ }^{\mathrm{g}}$ \\ ${ }^{a}$ Research Applications Laboratory, National Center for Atmospheric Research, Boulder, Colorado \\ ${ }^{\mathrm{b}}$ Houston Department of Health and Human Services, Houston, Texas \\ ${ }^{\mathrm{c}}$ Center for Statistics and Visualization, University of Denver, Denver, Colorado \\ ${ }^{\mathrm{d}}$ University of Colorado at Denver, Denver, Colorado \\ e The Climate Corporation, San Francisco, California \\ ${ }^{\mathrm{f}}$ Colorado Department of Labor and Employment, Denver, Colorado \\ ${ }^{\mathrm{g}}$ Toronto Public Health, Toronto, Ontario, Canada
}

(Manuscript received 8 November 2016, in final form 10 July 2017)

\begin{abstract}
Extreme heat is the leading cause of weather-related mortality in the United States, suggesting the necessity for better understanding population vulnerability to extreme heat. The work presented here is part of a larger study examining vulnerability to extreme heat in current and future climates [System for Integrated Modeling of Metropolitan Extreme Heat Risk (SIMMER)] and was undertaken to assess Houston, Texas, residents' adaptive capacity to extreme heat. A comprehensive, semistructured survey was conducted by telephone at 901 households in Houston in 2011. Frequency and logistic regression analyses were conducted. Results show that $20 \%$ of the survey respondents reported heat-related symptoms in the summer of 2011 despite widespread air conditioning availability throughout Houston. Of those reporting heat-related symptoms experienced in the home $(n=56)$, the majority could not afford to use air conditioning because of the high cost of electricity. This research highlights the efficacy of community-based surveys to better understand adaptive capacity at the household level; this survey contextualizes population vulnerability and identifies more targeted intervention strategies and adaptation actions.
\end{abstract}

\section{Introduction}

Extreme heat is a leading cause of weather-related human mortality in the United States (NOAA 2016) and many countries worldwide (Hajat and Kosatsky 2010). Heat-related negative health outcomes typically occur when daily temperatures exceed a normal range for a given climate, local setting, and availability of adaptations (Patz et al. 2005; McMichael et al. 2006). Societal vulnerability often determines the magnitude and the distribution of negative impacts of extreme heat on human health (Harlan et al. 2006; Johnson et al. 2009; Wilhelmi and Hayden 2010; Uejio et al. 2011; Johnson

Supplemental information related to this paper is available at the Journals Online website: https://doi.org/10.1175/WCASD-16-0125.s1.

Corresponding author: Mary H. Hayden, mhayden@ucar.edu et al. 2012; Harlan et al. 2014; Rosenthal et al. 2014). Climate change, urbanization, and demographic trends (e.g., ageing population) suggest that extreme heat will persist as a major hazard in the United States (Field et al. 2012; Oleson et al. 2015; Jones et al. 2015), disproportionately affecting the most vulnerable populations. For both public health and climate adaptation planning, it is essential to better understand which populations are most vulnerable and how to best reduce the negative health impacts of extreme heat exposure to these populations (Balbus and Malina 2009).

Climate adaptation will require a range of behavioral and technological modifications to reduce exposure to extreme heat. As cities develop, climate adaptation and hazard risk management plans (Harlan and Ruddell 2011; Boeckmann and Rohn 2014), characterizing urban vulnerability and especially adaptive capacity among the most vulnerable residents, will help identify strategies for heat hazard mitigation and climate adaptation. 
Research on adaptive capacity contributes to vulnerability research, which, in broad terms, examines the nature and fabric of a society's ability to prepare for and recover from natural hazards (Adger et al. 2004; Cutter and Finch 2008; Cutter et al. 2010; Wilhelmi and Hayden 2010; Hayden et al. 2011). Although the spectrum of vulnerability can be considered from a population level to an individual level, in this paper we focus on better understanding adaptive capacity at a household level. Societal vulnerability in general and lack of adaptive capacity in particular are both related to social inequalities, and earlier research indicates that there is unequal distribution of heat-related health risks based on both physical and social parameters. This suggests the need to go beyond examining demographic data to assess vulnerability to extreme heat by incorporating social and behavioral factors into vulnerability research (Adger et al. 2004; Wilhelmi et al. 2012).

After Wilhelmi and Hayden (2010), we characterize adaptive capacity to extreme heat as a function of knowledge, attitudes, and practices (KAP) at the household level. This includes household financial resources, access to public services and extreme heat risk reduction programs, and availability of strong social networks. An important focus of researching adaptive capacity is understanding existing access (or lack of access) to resources to cope with hazardous events (Blaikie et al. 1994; Wilhelmi and Hayden 2010). In this light, a range of factors including household air conditioning as an adaptive response need to be studied, particularly as a consideration of whether installation of air conditioning presents a feasible, equitable, and sustainable solution (Maller and Strengers 2011) for urban households. Differential capacity to cope with extreme heat exists even within similar demographic strata, as shown in previous research on vulnerability to extreme heat in Phoenix, Arizona. This may include information on tree canopy and impervious surfaces, two factors that may reduce the impacts of extreme heat but may be lacking in low-income neighborhoods (Harlan et al. 2006; Harlan et al. 2013, 2014) or may focus strictly on adaptive capacity at a household level (Hayden et al.2011). The framework developed by Wilhelmi and Hayden (2010) advocates for household-level research to ensure that interventions are targeted so that sustainable, community-driven responses are feasible. In the review of recent literature, Boeckmann and Rohn (2014) identified several studies that improve our understanding of adaptive capacity to extreme heat through surveys to determine heat risk awareness, effectiveness of heat warning systems, and protective behaviors during heat waves. Our case study expands on this topic by investigating household-level adaptive capacity to extreme heat in Houston, Texas. This research is part of a larger interdisciplinary project that examined vulnerability to extreme heat in current and future climates [System for Integrated Modeling of Metropolitan Extreme Heat Risk (SIMMER); Heaton et al. 2014; Heaton et al. 2015; Wilhelmi and Hayden 2016]. The objectives of the case study presented here were 1) to characterize the adaptive capacity to extreme heat in diverse urban households in Houston, Texas, and 2) to improve our understanding of how different socioeconomic disparities and behavioral factors interact to compound vulnerability to extreme heat. We approached these objectives through examining knowledge, attitudes, and practices; access to resources; awareness and use of city heat risk reduction programs; and existing social capital. Section 2 of this article describes the methodology employed in this study, including survey design, sampling strategy, and the data analysis. Section 3 presents the results of the survey, and section 4 offers a discussion of the main findings. We address study limitations in section 5 and summarize the key findings from this study and their implications in section 6 .

\section{Methods}

\section{a. Study site}

Houston, Texas, located in Harris County, is the largest city in Texas and the fourth largest in the United States with a 2010 population of 2.1 million (U.S. Census Bureau 2016). Houston has a fast-growing, multicultural population with over 145 languages spoken (Kriel 2015). It is located on the gulf coastal plain in southern Texas and has a humid subtropical (Köppen Cfa) climate with summer temperatures often above $32^{\circ} \mathrm{C}$ (NOAA 2013). In 2011, Houston experienced the hottest summer on record with temperatures regularly topping $37^{\circ} \mathrm{C}$. August 2011 had 24 days with ambient temperatures over $37^{\circ} \mathrm{C}$, making it the hottest month on record (Hoerling et al. 2013). Furthermore, a study by Zhang et al. (2015) found a significant association between extreme heat in the summer of 2011 and a higher than normal number of emergency department visits, particularly among the elderly. Coupled with Houston's growth, diversity, and hot summer climate come challenges such as an aging population, educational and income disparities, and a projected increase in high heat stress days and nights (Oleson et al. 2015). Current and future heat health risks in Houston (Heaton et al. 2014; Conlon et al. 2016; Marsha et al. 2016) call for a better understanding of the population's capacity to cope with extreme heat and to adapt to potentially more frequent and intense summer heat waves. 


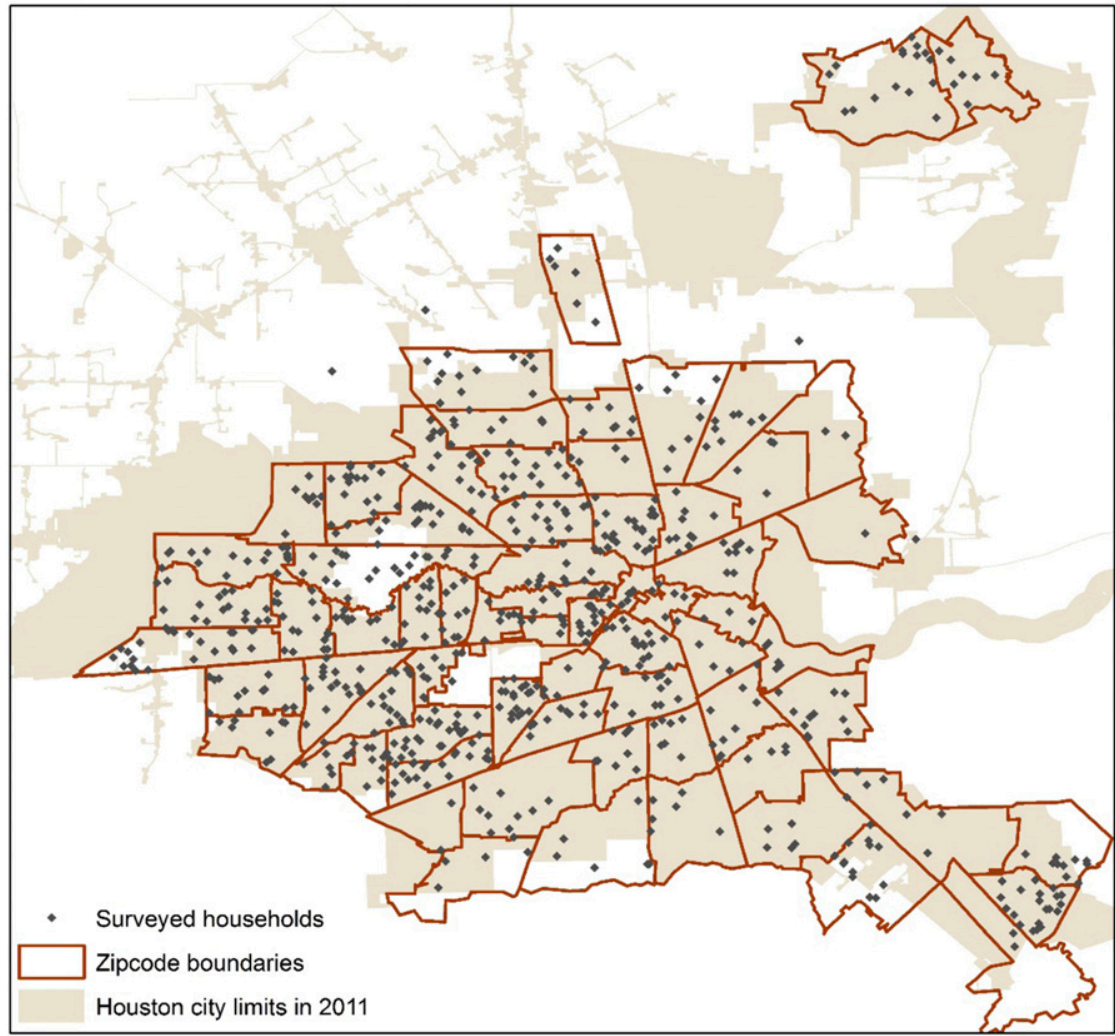

FIG. 1. Distribution of georeferenced households $(n=767)$ surveyed in Houston, Texas, in 2011.

\section{b. Survey development}

A semistructured interview protocol, based on Hayden et al. (2011), was adapted for use in Houston in collaboration with the Houston Department of Health and Human Services in the aftermath of extreme heat in the summer of 2011. The survey sought to understand Houston residents' adaptive capacity to extreme heat including knowledge, attitudes, and practices; participants' social capital; and their access to resources as well as knowledge of the availability of heat stress prevention programs. The survey consisted of both closed-ended and open-ended questions. Closed-ended questions were used to generate quantitative data, while openended questions helped to contextualize closed-ended responses and allow respondents to describe their experiences and views in greater depth. The survey was pretested in English and Spanish with multiple residents of the Houston area in July of 2011 and revised prior to implementation.

\section{c. Sampling strategy}

The random digit dial (RDD) survey was administered via telephone by Princeton Data Source, LLC
(PDS 2016), a survey data collection company; 901 adults living in Houston were surveyed from 31 October to 17 November 2011. This time period was chosen to ensure maximum recall of participants' experiences during the summer of 2011. Telephone surveys were conducted in English and Spanish, based on the respondent's preference. Calls were staggered over times of the day, and days of the week, to maximize the chance of making contact with a wide variety of respondents.

Three samples were used for data collection. All three samples covered the entire Houston area as defined by 71 ZIP codes (Fig. 1). The first sample was an equal probability sample of RDD phone numbers drawn from landline telephone exchanges that serve the listed ZIP codes. This sample was drawn using standard listassisted random digit dialing methodology from exchanges that serve the Houston area. The second sample was an equal probability landline listed sample drawn from the same ZIP code list noted above but was designed to garner information from unlisted numbers. The third sample was an equal probability RDD cellular phone sample drawn from rate centers that serve the Houston area. Out of 901 survey respondents, 767 participants provided the geolocation of their households or 
nearest cross streets. These households are shown in Fig. 1. Although not all 901 surveyed households are represented on the map, Fig. 1 shows the general distribution of households across Houston and the spatial pattern of this georeferenced subsample, which is representative of the overall sample.

\section{d. Statistical analysis}

Analyses were performed using SPSS software (version 21, IBM). Frequency analyses were conducted to provide descriptions of demographic characteristics and quantify participants' responses to survey questions. Logistic regression analyses were run to evaluate whether significant differences existed between different groups' responses to questions with binary responses. All analyses were unweighted. To maintain consistency in the analysis, each of these logistic regressions used the following explanatory variables: gender, income (less than $\$ 30,000$, between $\$ 30,000$ and $\$ 75,000$, and greater than $\$ 75,000$ ), education (did not graduate high school, high school graduate, and 4 or more years of college), age (30 years or less, 31-45, 4665, and greater than 65), employment status (employed, unemployed, and retired), race (Caucasian, AfricanAmerican, Hispanic, or other), language (English spoken at home or no English spoken at home), and homeownership.

\section{Results}

\section{a. Demographics}

Slightly more than half of the 901 respondents were female $(56.9 \%)$. The majority of the participants reported their race as Caucasian $(46.7 \%)$, while $26.6 \%$ of participants reported African-American race/ethnicity, 20.9\% reported Hispanic race/ethnicity, 4\% identified as Asian, $0.8 \%$ identified as American Indian/Alaska Native, and $0.2 \%$ identified as Native Hawaiian/Pacific Islander. Most of the sample's respondents had lived in the Houston area for more than 20 years $(63.9 \%)$, and over half of respondents were homeowners $(58.9 \%)$. There was a bimodal distribution of yearly household income; nearly half $(45.8 \%)$ of those respondents who reported their income indicated that it was less than $\$ 40,000$ a year, while $19.2 \%$ reported that their income was greater than $\$ 100,000$ a year. The sample was relatively well educated; $41.9 \%$ reported completing at least 4 years of college. Fully $47.4 \%$ of the sample was unemployed or retired. English was spoken by $88.3 \%$ of participants, and $11.7 \%$ spoke no English at home. An overview of the survey participants' demographics can be found in Table 1 .

\section{b. Awareness of heat risks}

A crucial first step in mitigating heat-related illness is increasing a population's awareness of the associated symptoms [Centers for Disease Control and Prevention (CDC) 2012]. The most commonly reported symptoms among the study participants $(n=894)$ were dizziness $(35.3 \%)$, fainting (22\%), fatigue $(18.9 \%)$, nausea/ vomiting $(18.2 \%)$, headaches $(14.9 \%)$, and, finally, intense thirst $(9.7 \%)$. A number of respondents indicated heavy sweating $(16.3 \%)$ as a symptom of too much heat exposure. Participants also mentioned clammy skin $(8.7 \%)$, dry mouth $(8.6 \%)$, weak/rapid pulse $(6.9 \%)$, and confusion $(6.2 \%)$ as potential symptoms; $14 \%$ of participants reported that they did not know any symptoms of heat-related illness.

The results of the logistic regression analysis on the knowledge of common symptoms are summarized in Table 2. Overall, the model is a good fit $(p=0.0001)$ with income $(p=0.009)$ and age $(p=0.008)$ being significant covariates. The odds of not knowing symptoms were almost 3 times greater for those who make less than $\$ 30,000 \mathrm{yr}^{-1}$ than for those who make over $\$ 75,000$ (95\% CI $=1.41$ to 5.55 ). Further, those over the age of 65 are significantly less likely to know the most common symptoms of heat-related illness.

Respondents were asked where they felt most at risk for heat-related illness; most $(92.1 \%)$ felt at highest risk outdoors. Of those who felt most at risk outdoors, $19.1 \%$ reported working outdoors.

\section{c. Sources of information about heat}

A majority of respondents $(87.7 \%)$ recalled having heard excessive heat warnings or advisories, likely because of the record-setting heat Houston experienced during the summer of 2011. Of participants who recalled hearing the advisories, the primary source of heatrelated information was from local television $(80.2 \%)$. Other sources cited by participants included radio $(34.7 \%)$ and newspapers $(15.8 \%)$.

Results from the logistic regression analysis are displayed in Table 3. Overall, the model is a good fit ( $p=$ $0.002)$, with gender $(p=0.048)$, education $(p=0.051)$, and age $(p<0.0001)$ being significant covariates. Those who were significantly more likely to recall hearing the heat advisories were between the ages of 31 and 65 . Males were 1.7 times less likely to recall hearing a heat advisory ( $p=0.048 ; 95 \% \mathrm{CI}=1.01-2.77)$ than females.

When asked where they received information about preventing heat-related illness, $66.6 \%$ of all respondents mentioned Houston local television stations. The Internet $(29.5 \%)$, radio $(26.9 \%)$, cable television $(25.9 \%)$, newspapers $(24.3 \%)$, and the Weather Channel (21.7\%) 
TABLE 1. Demographic characteristics of the survey participants.

\begin{tabular}{|c|c|c|c|}
\hline \multicolumn{4}{|c|}{ Sociodemographics } \\
\hline Variable & Frequency & Percent & 5-yr ACS (2006-10) \\
\hline \multicolumn{4}{|l|}{$\operatorname{Sex}(n=901)$} \\
\hline Male & 388 & $43.1 \%$ & $50.1 \%$ \\
\hline Female & 513 & $56.9 \%$ & $49.9 \%$ \\
\hline \multicolumn{4}{|l|}{ Race/ethnicity $(n=872)$} \\
\hline Caucasian & 407 & $46.7 \%$ & $54.8 \%$ \\
\hline Hispanic & 182 & $20.9 \%$ & $42.4 \%$ (any race) \\
\hline African-American & 232 & $26.6 \%$ & $24.4 \%$ \\
\hline Asian & 35 & $4.0 \%$ & $5.9 \%$ \\
\hline Native Hawaiian/Pacific Islander & 2 & $0.2 \%$ & $0.1 \%$ \\
\hline American Indian/Alaska Native & 7 & $0.8 \%$ & $0.4 \%$ \\
\hline Other & 7 & $0.8 \%$ & $14.4 \%$ \\
\hline \multicolumn{4}{|l|}{ Language $(n=901)$} \\
\hline English & 796 & $88.3 \%$ & $54.7 \%$ \\
\hline Spanish & 241 & $26.7 \%$ & $37.2 \%$ \\
\hline French & 20 & $2.2 \%$ & $\mathrm{~b}$ \\
\hline Chinese & 13 & $1.4 \%$ & $\mathrm{~b}$ \\
\hline Vietnamese & 5 & $0.6 \%$ & $\mathrm{~b}$ \\
\hline Other & 43 & $4.8 \%$ & $1.1 \%$ \\
\hline \multicolumn{4}{|l|}{ Income $(n=690)$} \\
\hline Less than $\$ 10,000$ & 103 & $13.4 \%$ & $8.7 \%$ \\
\hline$\$ 10,000-20,000$ & 94 & $12.3 \%$ & $13.3 \%$ \\
\hline$\$ 20,000-30,000$ & 98 & $12.8 \%$ & $13.1 \%$ \\
\hline$\$ 30,000-40,000$ & 56 & $7.3 \%$ & $11.7 \%$ \\
\hline$\$ 40,000-50,000$ & 44 & $5.7 \%$ & $9.3 \%$ \\
\hline$\$ 50,000-75,000$ & 77 & $10.0 \%$ & $16.6 \%$ \\
\hline$\$ 75,000-100,000$ & 71 & $9.3 \%$ & $9.4 \%$ \\
\hline More than $\$ 100,000$ & 147 & $19.2 \%$ & $17.9 \%$ \\
\hline \multicolumn{4}{|l|}{ Education $(n=890)$} \\
\hline No schooling & 13 & $1.5 \%$ & $2.5 \%$ \\
\hline Grades $1-8$ & 51 & $5.7 \%$ & $11.9 \%$ \\
\hline Grades 9-11 & 82 & $9.2 \%$ & $9.5 \%$ \\
\hline Grade 12/GED & 166 & $18.6 \%$ & $25.4 \%$ \\
\hline College $1-3$ years & 204 & $22.9 \%$ & $22.5 \%$ \\
\hline College $4+$ years & 374 & $41.9 \%$ & $28.2 \%$ \\
\hline \multicolumn{4}{|l|}{ Homeownership $(n=893)$} \\
\hline Own & 527 & $58.9 \%$ & $46.6 \%$ \\
\hline Rent & 333 & $37.2 \%$ & $53.4 \%$ \\
\hline Other arrangement & 33 & $3.7 \%$ & - \\
\hline \multicolumn{4}{|l|}{ Lived in Houston area $(n=901)$} \\
\hline Less than 1 year & 14 & $1.6 \%$ & c \\
\hline 1 to 4 years & 81 & $9.0 \%$ & $\mathrm{c}$ \\
\hline 5 to 9 years & 82 & $9.1 \%$ & $\mathrm{c}$ \\
\hline 10 to 19 years & 148 & $16.4 \%$ & c \\
\hline 20 years or more & 576 & $63.9 \%$ & c \\
\hline \multicolumn{4}{|l|}{ Employed $(n=884)$} \\
\hline Yes & 473 & $53.5 \%$ & $62.5 \%$ \\
\hline No (unemployed or retired) & 411 & $46.5 \%$ & d \\
\hline
\end{tabular}

${ }^{a}$ All demographic data derived from 2010 American Community Survey (ACS) 5 yr (2006-10).

${ }^{\mathrm{b}}$ French, Chinese, Vietnamese not reported individually in ACS data (see excel sheet).

${ }^{\mathrm{c}}$ No data for how long people had been living in Houston.

${ }^{\mathrm{d}}$ Unemployed: $5.4 \%$, Not in Labor Force: $32 \%$. Estimates of the retired population are not reported with employment information. 
TABLE 2. Binary logistic regression on the knowledge of common symptoms (know most common $=0$, do not know most common $=1$ ).

\begin{tabular}{|c|c|c|c|c|c|}
\hline \multicolumn{6}{|c|}{ Binary logistic regression on the knowledge of common symptoms $($ know most common $=0$, do not know most common $=1)$} \\
\hline & Parameter & Estimate & $\begin{array}{l}\text { Wald chi } \\
\text { square }\end{array}$ & $\begin{array}{l}\text { Sig. pr }>\text { chi } \\
\text { square }\end{array}$ & Odds ratio estimates \\
\hline \multicolumn{6}{|c|}{ Sociodemographic factors } \\
\hline 1.657 & $\begin{array}{l}\text { Male } \\
\text { Female }\end{array}$ & -0.196 & 0.968 & 0.325 & 0.822 \\
\hline Income $\left(X^{2}=9.527, p=0.009\right)$ & $\begin{array}{l}\text { Less than } 30000 \\
30000-75000 \\
\text { Greater than } 75000 \text { (reference) }\end{array}$ & $\begin{array}{l}1.027 \\
0.359\end{array}$ & $\begin{array}{l}8.614 \\
1.368\end{array}$ & $\begin{array}{l}0.003 \\
0.242\end{array}$ & $\begin{array}{l}2.793 \\
1.432\end{array}$ \\
\hline Education $\left(X^{2}=0.220, p=0.896\right)$ & $\begin{array}{l}\text { Did not graduate high school } \\
\text { High school graduate } \\
4 \text { or more years of } \\
\text { college (reference) }\end{array}$ & $\begin{array}{l}-0.125 \\
-0.124\end{array}$ & $\begin{array}{l}0.134 \\
0.231\end{array}$ & $\begin{array}{l}0.714 \\
0.631\end{array}$ & $\begin{array}{l}0.882 \\
0.884\end{array}$ \\
\hline Age $\left(X^{2}=11.537, p=0.009\right)$ & $\begin{array}{l}30 \text { years or less } \\
31-45 \\
46-65 \\
\text { Greater than } 65 \text { (reference) }\end{array}$ & $\begin{array}{l}-1.446 \\
-1.930 \\
-1.678\end{array}$ & $\begin{array}{r}5.991 \\
10.711 \\
8.659\end{array}$ & $\begin{array}{l}0.014 \\
0.001 \\
0.003\end{array}$ & $\begin{array}{l}0.236 \\
0.145 \\
0.187\end{array}$ \\
\hline Employed $\left(X^{2}=2.636, p=0.268\right)$ & $\begin{array}{l}\text { Employed } \\
\text { Unemployed } \\
\text { Retired (reference) }\end{array}$ & $\begin{array}{l}0.907 \\
1.020\end{array}$ & $\begin{array}{l}2.355 \\
2.696\end{array}$ & $\begin{array}{l}0.125 \\
0.101\end{array}$ & $\begin{array}{l}2.478 \\
2.772\end{array}$ \\
\hline Race $\left(X^{2}=2.535, p=0.469\right)$ & $\begin{array}{l}\text { Other } \\
\text { Hispanic } \\
\text { African-American } \\
\text { Caucasian (reference) }\end{array}$ & $\begin{array}{l}0.607 \\
0.168 \\
0.283\end{array}$ & $\begin{array}{l}2.050 \\
0.283 \\
1.102\end{array}$ & $\begin{array}{l}0.152 \\
0.595 \\
0.294\end{array}$ & $\begin{array}{l}1.835 \\
1.183 \\
1.328\end{array}$ \\
\hline Language & $\begin{array}{l}\text { English spoken } \\
\text { No English spoken }\end{array}$ & -0.333 & 1.128 & 0.288 & 0.717 \\
\hline Homeownership & $\begin{array}{l}\text { Own home } \\
\text { Do not own home }\end{array}$ & -0.251 & 1.073 & 0.300 & 0.778 \\
\hline
\end{tabular}

were also cited. More than a quarter of participants $(27.5 \%)$ indicated that they received heat-related illness information by word of mouth; evaluation of qualitative responses indicates that "word of mouth" includes respondents' friends, family, and coworkers.

\section{d. Experience with extreme heat in 2011}

The survey responses indicated that the presence of air conditioning did not always prevent heat-related illness. Almost $87 \%$ of survey participants had central air conditioning in their homes; however, $36.9 \%$ of all respondents said that they had felt too hot in their home during the summer of 2011. Demographic factors associated with participants feeling too hot at home included not being a homeowner and being female. Homeowners were 1.7 times more likely to report feeling comfortable in their homes ( $p=0.016 ; 95 \% \mathrm{CI}=1.11$ to 2.61 ), and males were 1.5 times more likely to report feeling comfortable in their homes $(p=0.028 ; 95 \% \mathrm{CI}=1.04$ to 2.09).

Participants were also asked, "This past summer, did you have symptoms such as fainting, rapid heartbeat, hallucinations, confusion, dizziness, or muscle cramps that you believe were heat-related?" Almost one-fifth of respondents (19.8\%) experienced some symptoms; of those experiencing symptoms, $74.7 \%$ experienced symptoms more than once, and $31.5 \%$ reported experiencing symptoms inside their homes. Of the 56 respondents who experienced symptoms in the home, $53(94.6 \%)$ used air conditioning (central air and/or window units).

The logistic regression model for those who experienced heat-related symptoms was a good fit $(p<$ $0.0001)$, with education $(p=0.026)$ and homeownership $(p=0.038)$ being significant. Those reporting less than 4 years of college were significantly more likely to have experienced heat-related illness, and those who did not graduate from high school had an even greater risk. Those who owned their homes were 1.7 times more likely to have had no symptoms of heat-related illness ( $p=0.038 ; 95 \% \mathrm{CI}=1.03-2.84)$ than those who rented their homes.

Additionally, respondents were asked to self-report whether their health at the time of the survey was good, fair, or poor. A second logistic regression model was fit using health status, knowing neighbors, and knowledge 
TABLE 3. Binary logistic regression on the recollection of excessive heat warnings (yes $=0$, no).

\begin{tabular}{|c|c|c|c|c|c|}
\hline \multicolumn{6}{|c|}{ Binary logistic regression on the recollection of excessive heat warnings $($ yes $=0$, no $=1$ ) } \\
\hline & Parameter & Estimate & Wald chi square & $\begin{array}{l}\text { Sig. pr }>\text { chi } \\
\text { square }\end{array}$ & $\begin{array}{c}\text { Odds ratio } \\
\text { estimates }\end{array}$ \\
\hline \multicolumn{6}{|c|}{ Sociodemographic factors } \\
\hline Sex & $\begin{array}{l}\text { Male } \\
\text { Female }\end{array}$ & 0.512 & 3.916 & 0.048 & 1.669 \\
\hline Income $\left(X^{2}=1.304, p=0.521\right)$ & $\begin{array}{l}\text { Less than } 30000 \\
30000-75000 \\
\text { Greater than } 75000 \text { (reference) }\end{array}$ & $\begin{array}{l}-0.503 \\
-0.336\end{array}$ & $\begin{array}{l}1.212 \\
0.794\end{array}$ & $\begin{array}{l}0.271 \\
0.373\end{array}$ & $\begin{array}{l}0.605 \\
0.715\end{array}$ \\
\hline Education $\left(X^{2}=5.950, p=0.051\right)$ & $\begin{array}{l}\text { Did not graduate high school } \\
\text { High school graduate } \\
4 \text { or more years of college } \\
\text { (reference) }\end{array}$ & $\begin{array}{r}0.859 \\
-0.080\end{array}$ & $\begin{array}{l}3.435 \\
0.053\end{array}$ & $\begin{array}{l}0.064 \\
0.817\end{array}$ & $\begin{array}{l}2.360 \\
0.923\end{array}$ \\
\hline Age $\left(X^{2}=21.389, p<0.0001\right)$ & $\begin{array}{l}30 \text { years or less } \\
31-45 \\
46-65 \\
\text { Greater than } 65 \text { (reference) }\end{array}$ & $\begin{array}{l}-0.688 \\
-1.602 \\
-2.023\end{array}$ & $\begin{array}{r}1.226 \\
6.359 \\
10.559\end{array}$ & $\begin{array}{l}0.268 \\
0.012 \\
0.001\end{array}$ & $\begin{array}{l}0.503 \\
0.201 \\
0.132\end{array}$ \\
\hline Employment $\left(X^{2}=4.346, p=0.114\right)$ & $\begin{array}{l}\text { Employed } \\
\text { Unemployed } \\
\text { Retired (reference) }\end{array}$ & $\begin{array}{l}1.386 \\
1.406\end{array}$ & $\begin{array}{l}4.294 \\
3.804\end{array}$ & $\begin{array}{l}0.038 \\
0.051\end{array}$ & $\begin{array}{l}3.998 \\
4.081\end{array}$ \\
\hline $\operatorname{Race}\left(X^{2}=1.500, p=0.682\right)$ & $\begin{array}{l}\text { Other } \\
\text { Hispanic } \\
\text { African-American } \\
\text { Caucasian (reference) }\end{array}$ & $\begin{array}{r}0.306 \\
-0.134 \\
0.254\end{array}$ & $\begin{array}{l}0.345 \\
0.104 \\
0.485\end{array}$ & $\begin{array}{l}0.557 \\
0.747 \\
0.486\end{array}$ & $\begin{array}{l}1.358 \\
0.874 \\
1.289\end{array}$ \\
\hline Language & $\begin{array}{l}\text { English spoken } \\
\text { No English spoken }\end{array}$ & -0.015 & 0.001 & 0.971 & 0.985 \\
\hline Homeownership & $\begin{array}{l}\text { Own home } \\
\text { Do not own home }\end{array}$ & -0.303 & 0.863 & 0.353 & 0.739 \\
\hline
\end{tabular}

of symptoms as covariates. Those who reported their health as either good or fair were almost 13 times more likely to report no heat-related symptoms compared to those who reported poor health $(p<0.0001 ; 95 \% \mathrm{CI}=$ 6.25-28.79).

As a follow on to the previous question, we asked participants what might prevent them from seeking medical care for heat-related symptoms; 122 (13.7\%) reported that certain factors such as lack of health insurance and cost of medical care might prevent them from seeking treatment (Fig. 2).

\section{e. Coping with extreme heat}

Strategies most commonly employed by participants to protect themselves from heat were staying indoors $(55.4 \%)$, drinking plenty of water $(53.9 \%)$, using an air conditioner $(25 \%)$, and avoiding outdoor activity (13.9\%; Fig. 3). A majority of the sample indicated that they alter their daily activities $(76.3 \%)$ when it gets too hot. The logistic regression model on changing outdoor activities was a good fit $(p<0.0001)$, with gender $(p=$ $0.001)$ and employment $(p=0.006)$ being significant and with education $(p=0.059)$ and income $(p=0.078)$ being marginally so. Males were almost 2 times less likely than females to change their outdoor activity plans ( $p=0.001 ; 95 \% \mathrm{CI}=1.33-2.94)$. Those who did not graduate from high school were 2.3 times less likely than college graduates to alter their daily activities in response to a heat warning ( $p=0.059 ; 95 \% \mathrm{CI}=1.14$ 4.57). Participants who earn less than $\$ 30,000 \mathrm{yr}^{-1}$ were also 2.3 times less likely than those who earn more than $\$ 75,000 \mathrm{yr}^{-1}$ to change their daily activities $(p=0.078$; $95 \% \mathrm{CI}=1.11-4.64)$.

Participants noted that when they changed their routines, they typically limited outdoor activities and/or engaged in outdoor activities early in the morning or late in the evening.

\section{f. Air conditioning as a coping mechanism}

Staying inside to avoid heat is only an effective strategy if the indoor environment is cooled. In Houston, most of our survey respondents reported having central air conditioning $(86.9 \%)$ and/or a window unit air conditioner $(14.5 \%)$. Other methods mentioned for cooling a house included fans $(44.1 \%)$ and awnings, shades, or shutters $(22.2 \%)$. Even though the full sample 


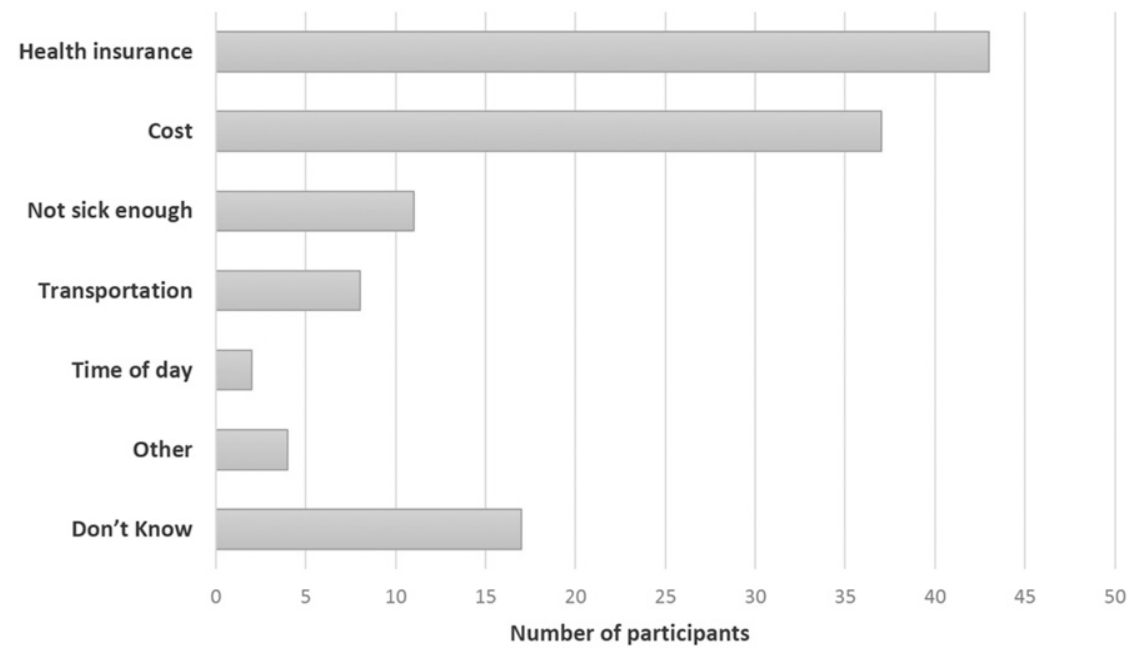

FIG. 2. Participants were asked, "Would anything prevent you from seeking medical care for heat-related symptoms?" Those who responded yes indicated the reasons for not seeking medical care for heat-related illness.

had high rates of air conditioning use, $14.4 \%$ of respondents reported that the cost of electricity prevented them from using their air conditioner (Table 4).

Participants were asked, "This past summer, did you have trouble paying your electric bill?" Almost onefourth $(24.5 \%)$ of respondents admitted having trouble paying their electric bill, with the average monthly bill provided by participants at $\$ 241.52$. The logistic regression model was a good fit $(p<0.0001)$ and income $(p<0.001)$, education $(p=0.003)$, age $(p=0.017)$, and race $(p=0.019)$ were significant. More specifically, those whose income was less than $\$ 75,000 \mathrm{yr}^{-1}$ had more difficulty paying their electric bill $(p<0.001)$. Additionally, those who had not graduated from high school $(p=0.003)$, those older than 30 years of age $(p=0.017)$, and those who reported race/ethnicity as AfricanAmerican $(p<0.019)$ had more difficulty paying their electric bill (Table 4).

\section{g. Awareness of programs and resources for coping with heat}

Paying the electric bill was problematic for some respondents; however, only $35.2 \%$ of all participants knew of special assistance programs offered by the local utility company to help pay electric bills. Based on the logistic regression model (overall $p<0.0001$ ), education ( $p=$ $0.05)$, age $(p=0.005)$, and race $(p=0.041)$ were significant. Those who had completed at least 4 years of

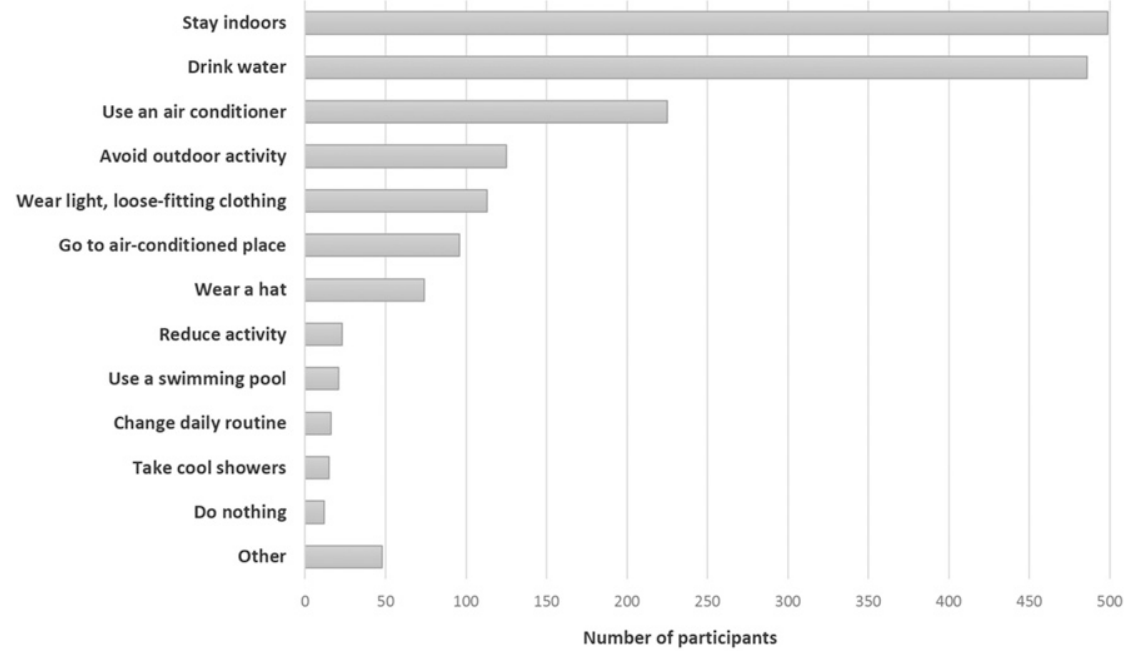

FIG. 3. Participants were asked, "During very hot weather, what steps do you take to protect yourself from the heat?" 
TABLE 4. Binary logistic regression on those that had trouble paying their electric bill (yes $=0$, no $=1$ ).

\begin{tabular}{|c|c|c|c|c|c|}
\hline \multicolumn{6}{|c|}{ Binary logistic regression on those that had trouble paying electric bill $($ yes $=0$, no $=1)$} \\
\hline & Parameter & Estimate & Wald chi square & $\begin{array}{l}\text { Sig. pr }>\text { chi } \\
\text { square }\end{array}$ & $\begin{array}{l}\text { Odds ratio } \\
\text { estimates }\end{array}$ \\
\hline \multicolumn{6}{|c|}{ Sociodemographic factors } \\
\hline Sex & $\begin{array}{l}\text { Male } \\
\text { Female }\end{array}$ & 0.270 & 1.657 & 0.198 & 1.310 \\
\hline Income $\left(X^{2}=20.639, p<0.0001\right)$ & $\begin{array}{l}\text { Less than } 30000 \\
30000-75000 \\
\text { Greater than } 75000 \text { (reference) }\end{array}$ & $\begin{array}{l}-1.855 \\
-1.213\end{array}$ & $\begin{array}{l}20.608 \\
10.851\end{array}$ & $\begin{array}{l}0.000 \\
0.001\end{array}$ & $\begin{array}{l}0.156 \\
0.297\end{array}$ \\
\hline Education $\left(X^{2}=11.377, p=0.003\right)$ & $\begin{array}{l}\text { Did not graduate high school } \\
\text { High school graduate } \\
4 \text { or more years of college } \\
\text { (reference) }\end{array}$ & $\begin{array}{l}-1.113 \\
-0.298\end{array}$ & $\begin{array}{l}9.982 \\
1.145\end{array}$ & $\begin{array}{l}0.002 \\
0.285\end{array}$ & $\begin{array}{l}0.329 \\
0.742\end{array}$ \\
\hline Age $\left(X^{2}=10.249, p=0.017\right)$ & $\begin{array}{l}30 \text { years or less } \\
31-45 \\
46-65 \\
\text { Greater than } 65 \text { (reference) }\end{array}$ & $\begin{array}{r}0.349 \\
-0.589 \\
-0.391\end{array}$ & $\begin{array}{l}0.166 \\
0.487 \\
0.217\end{array}$ & $\begin{array}{l}0.684 \\
0.485 \\
0.642\end{array}$ & $\begin{array}{l}1.418 \\
0.555 \\
0.676\end{array}$ \\
\hline Employment $\left(X^{2}=1.296, p=0.523\right)$ & $\begin{array}{l}\text { Employed } \\
\text { Unemployed } \\
\text { Retired (reference) }\end{array}$ & $\begin{array}{l}-0.347 \\
-0.594\end{array}$ & $\begin{array}{l}0.158 \\
0.443\end{array}$ & $\begin{array}{l}0.691 \\
0.506\end{array}$ & $\begin{array}{l}0.707 \\
0.552\end{array}$ \\
\hline Race $\left(X^{2}=9.947, p=0.019\right)$ & $\begin{array}{l}\text { Other } \\
\text { Hispanic } \\
\text { African-American } \\
\text { Caucasian (reference) }\end{array}$ & $\begin{array}{l}-0.497 \\
-0.332 \\
-0.846\end{array}$ & $\begin{array}{l}1.035 \\
1.036 \\
9.159\end{array}$ & $\begin{array}{l}0.309 \\
0.309 \\
0.002\end{array}$ & $\begin{array}{l}0.608 \\
0.717 \\
0.429\end{array}$ \\
\hline Language & $\begin{array}{l}\text { English spoken } \\
\text { No English spoken }\end{array}$ & -0.074 & 0.051 & 0.821 & 0.929 \\
\hline Homeownership & $\begin{array}{l}\text { Own home } \\
\text { Do not own home }\end{array}$ & 0.050 & 0.040 & 0.842 & 1.051 \\
\hline
\end{tabular}

college were over twice as likely as those who had not graduated from high school to be aware of assistance programs $(p=0.05 ; 95 \% \mathrm{CI}=1.13$ to 4.26$)$. Participants over 65 years of age were 3 times more likely to be aware of assistance programs than those under 30 years of age ( $p=0.005 ; 95 \% \mathrm{CI}=0.96$ to 9.67 ). Respondents who identified as Caucasian were twice as likely to be aware of assistance programs than those who identified as Hispanic ( $p=0.04 ; 95 \% \mathrm{CI}=1.06$ to 3.27 ) or AfricanAmerican ( $p=0.04 ; 95 \% \mathrm{CI}=1.07$ to 2.73$)$.

During heat emergencies when the City of Houston Heat Emergency Plan (City of Houston 2016) is activated, all public libraries and many multiservice centers are open to the public as cooling centers. These designated cooling centers and other city facilities provide an air-conditioned environment for residents who need to escape the effects of the heat. To gauge the population's knowledge of these cooling centers, participants were asked, "Do you know what a cooling center is?" Less than half (44\%) of respondents indicated that they knew what a cooling center was. Of those respondents who knew what a cooling center was, $45.3 \%$ were familiar with the location of a center, and $7 \%$ had used one. Of the 332 respondents who reported feeling too hot inside their home, $120(36.1 \%)$ knew what a cooling station was. Most respondents noted that they had never felt the need to use a cooling center because they had air conditioning in their homes.

\section{h. Social networks and social capital}

To better understand the potential role of social networks and social capital for reducing vulnerability to extreme heat, participants were asked a series of questions about their neighbors and neighborhoods. Participants were asked, "Thinking about your neighbors; would you say you know all, some, or none of them?" A small percentage of respondents $(17.4 \%)$ reported knowing all of their neighbors, while $71.3 \%$ reported knowing some of their neighbors. The logistic model suggests that those who knew at least some of their neighbors were more likely to be homeowners $(p=$ $0.004)$. The participants who knew all or some of their neighbors $(88.7 \%)$ would feel comfortable asking a neighbor for help, and more than a third (34.3\%) reported checking on a neighbor when the weather was hot. Participants were also asked, "When the weather is 
very hot, do any of your family members, friends, or neighbors who don't live in this home check on you?" Of the respondents, $46 \%$ answered affirmatively. Almost $11 \%$ of the respondents knew none of their neighbors; of these, $25.8 \%$ lived alone. Additionally, $6 \%$ of respondents knew none of their neighbors and also were not members of either a religious or nonreligious organization.

Participants were asked, "Do you consider people in your neighborhood vulnerable to heat?" Of the respondents, $40.2 \%$ answered affirmatively. Of those who responded affirmatively, $89.4 \%$ knew at least some of their neighbors, $85.5 \%$ talk to their neighbors at least occasionally, and $79.3 \%$ would feel comfortable asking a neighbor for help.

Almost all of those surveyed (90.6\%) reported feeling safe in their neighborhoods, although the logistic regression model suggested that participants who lived in households with incomes greater than $\$ 75,000 \mathrm{yr}^{-1}$ were 5 times more likely to feel safe in their neighborhoods than those making less than $\$ 30,000 \mathrm{yr}^{-1}(p=0.03 ; 95 \%$ $\mathrm{CI}=1.44$ to 15.46$)$. Additionally, those who selfidentified as being in good to fair health $(p=0.009)$ and those who knew at least some of their neighbors $(p=0.014)$ reported feeling safe in their neighborhoods.

\section{Discussion}

Adaptive capacity at the household level is an understudied area in vulnerability research. Yet, knowledge about adaptive capacity can shape short-term responses to extreme heat, public health interventions, and longterm adaptation strategies. This study is one of the first systematic attempts to characterize adaptive capacity to extreme heat at the household level across a major metropolitan area. The population sample presented here represents a demographically broad range of urban residents in Houston, Texas.

Multiple demographic, socioeconomic, and behavioral factors may interact to compound vulnerability. In our study, during the summer of 2011, nonhomeowners, African-Americans and Hispanic/Latinos, those with household incomes less than $\$ 30,000 \mathrm{yr}^{-1}$, those who reported being in poor health, and those who were unemployed were significantly more likely to report heat stress and symptoms of heat-related illness. Those who reported poor health were significantly less likely to know their neighbors, be nonhomeowners, or feel safe in their neighborhoods, suggesting that they may be more vulnerable to heat-related illness.

Earlier studies have pointed to air conditioning as a present-day response to and a mechanism for adaptation to extreme heat under future climate change (Davis et al. 2003; Donaldson et al. 2003; Curriero et al. 2002; Braga et al. 2001), with a study by Barnett (2007) suggesting that risk of cardiovascular mortality from extreme heat had disappeared across 104 cities in the United States, most likely because of increased air conditioning usage. While these finding may lead to the assumption that air conditioning will alleviate negative health outcomes from extreme heat, other studies begin to question whether household air conditioning is a short-sighted approach to adaptation (Maller and Strengers 2011). Recent research presents evidence that warmer conditions due to a changing climate increase demand for air conditioning in cities. Increased use of air conditioners contributes to greenhouse gas emissions at the global scale and generates more heat at the local scale (Grimmond 2007; Lundgren and Kjellstrom 2013), thus increasing extreme heat exposure. In addition to challenges associated with warmer urban conditions and growing energy demand, research shows that household use of air conditioning may exacerbate existing inequalities among urban residents. Farbotko and Waitt (2011) demonstrated that low-income elderly residents in Australian households could not afford to use air conditioning without energy subsidies. Therefore, while air conditioning may provide effective and immediate relief from extreme heat, more sustainable approaches are needed to reduce extreme heat risk (Lundgren and Kjellstrom 2013).

Our study provides quantitative empirical evidence that despite an overall high percentage (almost $87 \%$ ) of the population in Houston with household central and/ or window unit air conditioners, many study participants still experienced symptoms of heat stress during the summer of 2011 . More than a third (37\%) of our study population expressed feeling too hot inside their homes despite air conditioning, and almost $20 \%$ of our participants reported experiencing heat-related symptoms during the summer of 2011. Those who experienced heat-related health problems also reported having access to air conditioning; however, those who rented their homes and were unemployed were unable to employ air conditioning to the extent needed to cool themselves adequately, either because the air conditioner was broken or they could not afford the cost of electricity to run the air conditioner. Additionally, $44 \%$ of our study population used fans in their homes; although, only a small number $(n=13)$ used fans exclusively to cool their homes. Earlier studies have shown the risks of using fans as a sole cooling mechanism, suggesting a need to continue to educate the public on this risk (Luber and McGeehin 2008). Caution is needed in drawing conclusions from this question as we did not ask respondents if the fans were in operation when they were 
not at home or if the windows were left open when fans were used.

Moreover, large-scale, power grid failures are not uncommon in the United States, leaving residents without air conditioning; one example occurred in Chicago during the heat wave of 1995 when the city set new records for power usage, resulting in selected grid failure, and 49000 people were left without power (Klinenberg 2002). In 2006, the United States experienced a severe heat wave and in selected areas, such as St. Louis, power was lost to over half a million customers (CNN 2006). In the summer of 2012, 4 million people in the eastern United States were left without power for a week in the aftermath of a derecho, an intense storm with winds in excess of $80 \mathrm{mph}$. The loss of power coupled with extreme heat resulted in 34 heat-related deaths (NOAA 2013). In Houston, power outages are common in the aftermath of hurricanes that often occur during very hot summer conditions, even in the absence of a heat wave. For example, on 13 September, Hurricane Ike left 2.9 million residents of the Houston-Galveston area without power; it took 16 days for power to be completely restored (Qiao and Vipulanandan 2009). These examples, coupled with our findings on air conditioner access in Houston, suggest that relying solely on residential air conditioning to combat heat exposure is not the ultimate answer to reducing population vulnerability to extreme heat.

In many cities, offering cooling centers is becoming a common practice for reducing the population's exposure to extreme heat (Berisha et al. 2017). During the European heat wave of 2003 , even $2-3$-h cooling sessions were shown to be beneficial to those who were vulnerable to the negative impacts of extreme heat, in particular, the elderly (Salagnac 2007). In Houston, only a small percentage of our study population was familiar with what a cooling center was, and of those, an even smaller percentage knew where one was located. This suggests that those populations identified as vulnerable might benefit from the provision of added information on the value of cooling centers, their hours of operations, directions for getting there using public or private transportation, and the importance of cooling oneself for at least a few hours per day during extreme heat events.

Most respondents surveyed in Houston reported hearing information about excessive heat advisories from their broadcasters on local TV, a phenomenon that has been reported previously with other extreme events such as hurricanes (Morss and Hayden 2010) as well as extreme heat (Sheridan 2006). In this study, 14\% of the population reported not knowing any symptoms of heat stress, and these were potentially among the most vulnerable including those who were unemployed and did not speak English as a first language. Additionally, 7\% of our participants reported using sunscreen as a means to protect themselves from heat stress, with Hispanic/ Latino populations significantly more likely to report this than other ethnic groups, suggesting a need to ensure that straightforward messages about risk reduction reach those who are potentially more vulnerable. In an earlier study undertaken in Phoenix, Arizona, Hayden et al. (2011) suggested that public health collaboration with local broadcast meteorologists to inform television audiences not only of an impending extreme heat event but also to provide information on symptoms of heat stress and risk reduction measures may be a step in proactively reducing vulnerability to heat.

\section{Study limitations}

Houston is one of the fastest-growing and most diverse cities in the United States; the demographic shift in the past decades has been well-documented by Klineberg (2017) in research conducted by the Kinder Institute. Demographic groups with less access to resources are somewhat underrepresented in the survey sample presented in this manuscript (Table 1). While weighting respondents is one way to address this issue, the results presented here are all unweighted. If a weighted response is considered, any results correlated with resource level (e.g., those related to income, thermostat setting, and employment) will be of greater magnitude and higher significance. The results presented here are not correlated to resource access and do not change in significance when a weighted analysis is considered instead.

\section{Conclusions}

As a part of a larger study on urban vulnerability to extreme heat in Houston, Texas, we conducted a household survey on population adaptive capacity to better understand a wide range of socioeconomic and health disparities. Our broad sample of urban households provided important information about the roles of knowledge, attitudes, and practices; access to resources; use and awareness of city heat risk reduction programs; and social capital. The survey identified nonhomeowners, AfricanAmerican and Hispanic/Latinos, those with incomes less than $\$ 30,000 \mathrm{yr}^{-1}$, those unemployed, and those in poor health to be most vulnerable to heat stress.

Additionally, findings indicate that these populations have little or no knowledge of the symptoms of heat stress nor do they know where the closest cooling center is. Often, financial barriers restricted the use of an air conditioner at home where the greatest number of 
participants reported symptoms of heat stress. These findings suggest heat mitigation and climate adaptation strategies have to include both technological and behavioral-/community-based options.

Acknowledgments. This work was supported by NASA Award NNX10AK79G. The National Center for Atmospheric Research is managed by the University Corporation for Atmospheric Research and is sponsored by the National Science Foundation. The authors also wish to thank Monica Childers for help with Houston GIS data, Cassandra O'Lenick for assistance with the demographic table, and the study respondents for participating.

\section{REFERENCES}

Adger, W. N., N. Brooks, G. Bentham, M. Agnew, and S. Eriksen, 2004: New indicators of vulnerability and adaptive capacity. Tyndall Centre for Climate Change Research Tech. Rep. 7, $128 \mathrm{pp}$.

Balbus, J. M., and C. Malina, 2009: Identifying vulnerable subpopulations for climate change health effects in the United States. J. Occup. Environ. Med., 51, 33-37, doi:10.1097/ JOM.0b013e318193e12e.

Barnett, A. G., 2007: Temperature and cardiovascular deaths in the US elderly: Changes over time. Epidemiology, 18, 369-372, doi:10.1097/01.ede.0000257515.34445.a0.

Berisha, V., and Coauthors, 2017: Assessing adaptation strategies for extreme heat: A public health evaluation of cooling centers in Maricopa County, Arizona. Wea. Climate Soc., 9, 71-80, doi: 10.1175/WCAS-D-16-0033.1.

Blaikie, P., T. Cannon, I. Davis, and B. Wisner, 1994: At Risk: Natural Hazards, People's Vulnerability, and Disasters. Routledge, $284 \mathrm{pp}$.

Boeckmann, M., and I. Rohn, 2014: Is planned adaptation to heatreducing heat-related mortality and illness? A systematic review. BMC Public Health, 14, 1112, doi:10.1186/1471-2458-14-1112.

Braga, A. L. F., A. Zanobetti, and J. Schwartz, 2001: The time course of weather-related deaths. Epidemiology, 12, 662-667, http://www.jstor.org/stable/3703185.

CDC, 2012: Recognizing, preventing, and treating heat-related illness: WB 2333. Accessed 1 September 2016, http://www.cdc. gov/nceh/hsb/extreme/heat_illness_training.htm.

City of Houston, 2016: Houston activates heat emergency planHoustonians urged to protect themselves against heat-related illnesses. Accessed 1 September 2016, http://www.houstontx. gov/health/NewsReleases/heatplan.html.

CNN, 2006: St. Louis struggles without power. CNN.com, 24 July, http://edition.cnn.com/2006/WEATHER/07/23/st.louis.blackout/.

Conlon, K., A. Monaghan, M. Hayden, and O. Wilhelmi, 2016: Potential impacts of future warming and land use changes on intra-urban heat exposure in Houston, Texas. PLoS One, 11, e0148890, doi:10.1371/journal.pone.0148890.

Curriero, F. C., K. S. Heiner, J. M. Samet, S. L. Zeger, L. Strug, and J. A. Patz, 2002: Temperature and mortality in 11 cities of the eastern United States. Amer. J. Epidemiol., 155, 80-87, doi:10.1093/aje/155.1.80.

Cutter, S. L., and C. Finch, 2008: Temporal and spatial changes in social vulnerability to natural hazards. Proc. Natl. Acad. Sci. USA, 105, 2301-2306, doi:10.1073/pnas.0710375105.
— C. G. Burton, and C. T. Emrich, 2010: Disaster resilience indicators for benchmarking baseline conditions. J. Homeland Secur. Emerg. Manage., 7, 1-22.

Davis, R. E., P. C. Knappenberger, P. J. Michaels, and W. M. Novicoff, 2003: Changing heat-related mortality in the United States. Environ. Health Perspect., 111, 1712, doi:10.1289/ehp.6336.

Donaldson, G. C., W. R. Keatinge, and S. Näyhä, 2003: Changes in summer temperature and heat-related mortality since 1971 in North Carolina, south Finland, and southeast England. Environ. Res., 91, 1-7, doi:10.1016/S0013-9351(02)00002-6.

Farbotko, C., and G. Waitt, 2011: Residential air-conditioning and climate change: Voices of the vulnerable. Health Promot. J. Aust., 22, 13-15.

Field, C. B., and Coauthors, Eds., 2012: Managing the Risks of Extreme Events and Disasters to Advance Climate Change Adaptation: Special Report of the Intergovernmental Panel on Climate Change. Cambridge University Press, 594 pp.

Grimmond, S., 2007: Urbanization and global environmental change: Local effects of urban warming. Geogr. J., 173, 83-88, doi:10.1111/j.1475-4959.2007.232_3.x.

Hajat, S., and T. Kosatsky, 2010: Heat-related mortality: A review and exploration of heterogeneity. J. Epidemiol. Community Health, 64, 753-760, doi:10.1136/jech.2009.087999.

Harlan, S. L., and D. M. Ruddell, 2011: Climate change and health in cities: Impacts of heat and air pollution and potential cobenefits from mitigation and adaptation. Curr. Opin. Environ. Sustainability, 3, 126-134, doi:10.1016/j.cosust.2011.01.001

- A. J. Brazel, L. Prashad, W. L. Stefanov, and L. Larsen, 2006: Neighborhood microclimates and vulnerability to heat stress. Soc. Sci. Med., 63, 2847-2863, doi:10.1016/ j.socscimed.2006.07.030.

, J. H. Declet-Barreto, W. L. Stefanov, and D. B. Petitti, 2013: Neighborhood effects on heat deaths: Social and environmental predictors of vulnerability in Maricopa County, Arizona. Environ. Health Perspect., 121, 197-204, doi:10.1289/ ehp. 1104625.

—, G. Chowell, S. Yang, D. B. Petitti, E. J. Morales Butler, B. L. Ruddell, and D. M. Ruddell, 2014: Heat-related deaths in hot cities: Estimates of human tolerance to high temperature thresholds. Int. J. Environ. Res. Public Health, 11, 3304-3326, doi:10.3390/ijerph110303304.

Hayden, M. H., H. Brenkert-Smith, and O. V. Wilhelmi, 2011: Differential adaptive capacity to extreme heat: A Phoenix, Arizona, case study. Wea. Climate Soc., 3, 269-280, doi:10.1175/WCAS-D-11-00010.1.

Heaton, M. J., and Coauthors, 2014: Characterizing urban vulnerability to heat stress using a spatially varying coefficient model. Spat. Spatio-Temporal Epidemiol., 8, 23-33, doi:10.1016/ j.sste.2014.01.002.

— S. R. Sain, A. J. Monaghan, O. V. Wilhelmi, and M. H. Hayden, 2015: An analysis of an incomplete marked point pattern of heat-related 911 calls. J. Amer. Stat. Assoc., 110, 123-135, doi:10.1080/01621459.2014.983229.

Hoerling, M., and Coauthors, 2013: Anatomy of an extreme event. J. Climate, 26, 2811-2832, doi:10.1175/JCLI-D-12-00270.1.

Johnson, D. P., J. S. Wilson, and G. C. Luber, 2009: Socioeconomic indicators of heat-related health risk supplemented with remotely sensed data. Int. J. Health Geogr., 8, 57, doi:10.1186/ 1476-072X-8-57.

, A. Stanforth, V. Lulla, and G. Luber, 2012: Developing an applied extreme heat vulnerability index utilizing socioeconomic and environmental data. Appl. Geogr., 35, 23-31, doi:10.1016/j.apgeog.2012.04.006. 
Jones, B., B. C. O'Neill, L. McDaniel, S. McGinnis, L. O. Mearns, and C. Tebaldi, 2015: Future population exposure to US heat extremes. Nat. Climate Change, 5, 652-655, doi:10.1038/ nclimate2631.

Klineberg, S. L., 2017: The Kinder Houston Area Community Survey: Thirty-six years of measuring responses to a changing America. Accessed on 23 August 2017, https://kinder.rice.edu/ uploadedFiles/Kinder_Institute_for_Urban_Research/HAS/ 2017\%20Kinder\%20Houston\%20Area\%20Survey\%20FINAL.pdf.

Klinenberg, E., 2002: Heat Wave: A Social Autopsy of Disaster in Chicago. University of Chicago Press, $328 \mathrm{pp}$.

Kriel, L., 2015: Just how diverse is Houston? 145 languages spoken here. Houston Chronical, 5 November, http://www. houstonchronicle.com/news/houston-texas/article/Houstoniansspeak-at-least-145-languages-at-home-6613182.php.

Luber, G., and M. McGeehin, 2008: Climate change and extreme heat events. Amer. J. Prev. Med., 35, 429-435, doi:10.1016/ j.amepre.2008.08.021.

Lundgren, K., and T. Kjellstrom, 2013: Sustainability challenges from climate change and air conditioning use in urban areas. Sustainability, 5, 3116-3128, doi:10.3390/su5073116.

Maller, C. J., and Y. Strengers, 2011: Housing, heat stress and health in a changing climate: Promoting the adaptive capacity of vulnerable households, a suggested way forward. Health Promot. Int., 26, 492-498, doi:10.1093/heapro/dar003.

Marsha, A., S. R. Sain, M. J. Heaton, A. J. Monaghan, and O. V. Wilhelmi, 2016: Influences of climatic and population changes on heat-related mortality in Houston, Texas, USA. Climatic Change, doi:10.1007/s10584-016-1775-1.

McMichael, A. J., R. E. Woodruff, and S. Hales, 2006: Climate change and human health: Present and future risks. Lancet, 367, 859-869, doi:10.1016/S0140-6736(06)68079-3.

Morss, R. E., and M. H. Hayden, 2010: Storm surge and "certain death": Interviews with Texas coastal residents following Hurricane Ike. Wea. Climate Soc., 2, 174-189, doi:10.1175/ 2010WCAS1041.1.

NOAA, 2013: The historic derecho of June 29, 2012. U.S. Department of Commerce/NOAA/NWS Service Assessment, 61 pp., https:/www.weather.gov/media/publications/assessments/ derecho12.pdf.

_ - 2016: Natural hazards statistics. National Weather Service Office of Climate, Water, and Weather Services, accessed 1 September 2016, http://www.nws.noaa.gov/om/hazstats.shtml.

Oleson, K. W., A. Monaghan, O. Wilhelmi, M. Barlage, N. Brunsell, J. Feddema, L. Hu, and D. F. Steinhoff, 2015: Interactions between urbanization, heat stress, and climate change. Climatic Change, 129, 525-541, doi:10.1007/ s10584-013-0936-8.

Patz, J. A., D. Campbell-Lendrum, T. Holloway, and J. A. Foley, 2005: Impact of regional climate change on human health. Nature, 438, 310-317, doi:10.1038/nature04188.

PDS, 2016: Princeton Data Source, LLC. Accessed 23 August 2017, http://www.princetondatasource.com/.

Qiao, W., and C. Vipulanandan, 2009: Modeling the power outage after Hurricane Ike. Proc. Texas Hurricane Center for Innovative Technology Conf., Houston, TX, University of Houston, 2 pp., http://hurricane.egr.uh.edu/sites/hurricane. egr.uh.edu/files/files/2009/wei.pdf.

Rosenthal, J. K., P. L. Kinney, and K. B. Metzger, 2014: Intraurban vulnerability to heat-related mortality in New York City, 1997-2006. Health Place, 30, 45-60, doi:10.1016/ j.healthplace.2014.07.014

Salagnac, J. L., 2007: Lessons from the 2003 heat wave: A French perspective. Build. Res. Inf., 35, 450-457, doi:10.1080/ 09613210601056554

Sheridan, S. C., 2006: Public perception and response to extreme heat. Bull. Amer. Meteor. Soc., 87, 884-886.

Uejio, C. K., O. V. Wilhelmi, J. S. Golden, D. M. Mills, S. P. Gulino, and J. P. Samenow, 2011: Intra-urban societal vulnerability to extreme heat: The role of heat exposure and the built environment, socioeconomics, and neighborhood stability. Health Place, 17, 498-507, doi:10.1016/j.healthplace.2010.12.005.

U.S. Census Bureau, 2016: Quick facts Houston City, Texas. U.S. Census Bureau, http://www.census.gov/quickfacts/table/ PST045215/4835000.

Wilhelmi, O., and M. H. Hayden, 2010: Connecting people and place: A new framework for reducing urban vulnerability to extreme heat. Environ. Res. Lett., 5, 014021, doi:10.1088/ 1748-9326/5/1/014021.

, and M. Hayden, 2016: Reducing vulnerability to extreme heat through interdisciplinary research and stakeholder engagement. Extreme Weather, Health, and Communities: Interdisciplinary Engagement Strategies, S. L. Steinberg and W. A. Sprigg, Eds., Springer, 165-186.

, A. de Sherbinin, and M. Hayden, 2012: Exposure to heat stress in urban environments: Current status and future prospects in a changing climate. Ecologies and Politics of Health, B. King and K. Crews, Eds., Routledge, 1-15.

Zhang, K., T.-H. Chen, and C. E. Begley, 2015: Impact of the 2011 heat wave on mortality and emergency department visits in Houston, Texas. Environ. Health, 14, 11, doi:10.1186/ 1476-069X-14-11. 